\title{
Inclusión Educativa en Fe y Alegría
}

Nelly Andrade ${ }^{10}$

Artículo recibido en abril y aprobado en mayo 2017

\section{Resumen}

La Inclusión Educativa en Fe y Alegría Ecuador nace como una reacción ante la vulneración de derechos, la discriminación, la exclusión y la marginación presente en el sistema educativo y social. Reflexionada y construida desde la teoría a la práctica y a partir de la investigación acción participativa, la inclusión se hace posible en el Movimiento sobre la base de la educación popular sustentada en cuatro pilares: ético, pedagógico, político y epistemológico, que en conjunto fortalecen una educación de calidad.

Ligado a ello, está la estructura organizacional que marca la línea de acción para hacer posible un proceso de inclusión educativa y social organizado, cuya articulación va desde los procesos gobernantes, pasando por los procesos sustantivos y adjetivos, más el horizonte de identidad y el accionar educativo con sus tres sistemas, siendo todos elementos esenciales que permiten la coherencia en la implementación de la inclusión educativa y evitan que se manifiesten las distintas formas de exclusión. Todo esto nos permite incidir en las políticas públicas, pero, ante todo, hacer válido el ejercicio de derechos como una práctica cotidiana.

Palabras claves: Inclusión educativa, educación inclusiva, educación popular, educación de calidad. 


\section{Educational Inclusion in Fe y Alegria}

\section{Abstract}

Educational Inclusion in Fe y Alegría Ecuador, arises from the reaction to the infringement of rights, discrimination, exclusion and marginalization throughout the educational and social system. Thoughtful and built from theory to practice and from action - research participation, inclusion becomes possible in the Movement, on the basis of popular education supported by four pillars: ethical, pedagogical, political and epistemological, which together strengthen an education of quality.

As part of this, there is the organizational structure that sets the course of action to make possible an organized educational and social inclusion process, whose articulation goes from the governing processes, passing through the substantive and adjective processes, and the "Horizonte de Identidad" (Skyline of Identity) and the educational action with its three systems, all essential that allow coherence in the implementation of educational inclusion and avoid manifesting the different forms of exclusion. All of this enables us to influence in public policies, but, above all, to make the exercise of rights valid as a daily practice.

Key words: Educational inclusion, inclusive education, popular education, quality education.

\section{Nuevas apuestas educativas}

"En sociedad cada individuo debe considerarse como un sentimiento y han de combinarse los sentimientos para hacer una conciencia social" (Simón Rodríguez, 1990, pag.34)

Fe y Alegría concibe la educación como una oportunidad de transformación social, política e individual que promueve la construc- 
ción de una nueva sociedad y la hace vida en los centros educativos. En ellos la comunidad desarrolla su sentido de pertenencia en espacios de libertad, participación y convivencia pacífica que posibilitan la vivencia de una ciudadanía activa combinando los sentimientos y las voces de protesta, las voces que proponen y aporten en conjunto ideas y sueños, entre otras cosas, para el reconocimiento de necesidades, fortalezas y saberes de cada individuo.

Desde esta lógica se fortalece el nuevo pensamiento que anduvo rodando desde hace aproximadamente diez años, respecto a hacer de los centros educativos escuelas para todas y todos. Esto contempla una educación de calidad direccionada a reducir la exclusión de grupos minoritarios del sistema educativo, identificar barreras que limitan el ejercicio de los derechos humanos y reconocer la diversidad como un aspecto esencial para cambiar estructuras. Por lo tanto, implica proponer nuevas políticas para que las prácticas del sistema educativo se direccionen con responsabilidad social, con una mirada y acciones de justicia, equidad, igualdad de oportunidades, reconocimiento de necesidades comunes, así como de la singularidad de cada estudiante respecto a sus "saberes y haceres"; invita a aplicar metodologías activas y críticas para un aprendizaje participativo y creativo, a reflexionar sobre los requerimientos pedagógicos para atender adecuadamente todas las necesidades presentes en el contexto escolar, también relacionadas con lo comunitario, lo social y lo familiar.

Así, se apuesta por una nueva educación, tomando como reto la Inclusión Educativa, la cual se fundamenta en el marco legal internacional, tratados y teorías globales, leyes, investigaciones y testimonios nacionales, además de planteamientos de la Federación Internacional de Fe y Alegría, con el fin de sensibilizar y organizar adecuadamente la atención sobre la diversidad a nivel educativo, social y comunitario y ofrecer a la sociedad una nueva mirada respecto a la forma de concebir la diferencia.

Se apuesta también por la construcción colectiva de una cultura inclusiva, a pesar de que es un proceso de continua tensión que nos inquieta y moviliza, ya que no existe receta alguna que responda por igual a las situaciones de cada contexto (Federación Internacional Fe y Alegría, 2013, pág 2). 
El Documento del XLIV Congreso Internacional de Fe y Alegría (2013) hace alusión a que la Inclusión Educativa es una nueva cultura que concentra características como:

- Ser colaborativa.

- Tener altas expectativas para educandos y educadores/as.

- Tener consenso en cuanto a valores.

- Tener habilidades para trabajar eficazmente con las diferencias presentes en el aula y en el centro educativo.

- Estar apoyada en un entorno ordenado y seguro.

- Reconocer y estimular a los educadores/as para que asuman una variedad de roles de liderazgo.

Elementos que están implícitos en el modelo de inclusión educativa que va adoptando Fe y Alegría Ecuador, contemplado en sus distintas etapas, en el marco teórico y en las opciones clave, como un camino hacia la promoción de una ideología inclusiva.

Para caminar hacia ese horizonte, es necesario seguir fortaleciendo el accionar educativo desde el ideario y la espiritualidad transformadora que contempla la construcción de hombres y mujeres capaces de humanizar y transformar las realidades, esto implica caminar hacia un horizonte de equidad, igualdad, justicia social, solidaridad y trabajo con y para los sectores excluidos (Federación Internacional Fe y Alegría, 2013, pág. 1).

Implica trabajar desde una espiritualidad trasformadora que contemple las realidades relacionadas con la inclusión educativa. Desde el trabajo en comunidad es posible generar estos cambios, poniendo de manifiesto los valores institucionales explicitados en el Horizonte Pedagógico Pastoral de Fe y Alegría que orientan todos los procesos del Movimiento: la justicia, donde cada persona tenga lo necesario para vivir dignamente; la solidaridad, que reconoce el rostro de otras personas para trabajar aunando esfuerzos; la participación, que da la posi- 
bilidad de aportar significativamente a la construcción de un proyecto social más justo; la equidad de género, que desde el reconocimiento de la particularidad de cada persona, busca la igualdad de derechos y de oportunidades más allá de roles predefinidos; la responsabilidad, comprendida como la seriedad, compromiso y vocación expuesta hacia las personas que crecen junto a nosotros; el respeto a la diversidad y la diferencia, que supone un trato igualitario, amable y cortés, como garantía absoluta de transparencia, libertad y reconocimiento del otro; la espiritualidad transformadora, que nos permite poner los pies sobre la tierra para partir del contexto en la búsqueda de un mundo mejor; la audacia, al asumir los riesgos en la búsqueda de mayores compromisos con la sociedad y el compromiso que nos lleva a poner con firmeza todas las capacidades al servicio de la misión y a alcanzar las metas y sueños. (Fe y Alegría Ecuador, 2016, pág. 15-16).

Por lo tanto, lo mencionado anteriormente inspira a reflexionar e invita a permanecer en el desafío de vivir el pleno ejercicio de la inclusión educativa; en palabras del padre Ignacio Suñol (en Fe y Alegría 2013), inclusión educativa se convierte en un reto, por lo que es importante poner atención a lo que sigue causando exclusión y marginación. Ante ello, el movimiento Fe y Alegría deberá estar presente en las diversas fronteras en "donde se acaba el asfalto de las oportunidades educativas" (Fe y Alegría, 2013, pág. 7).

Y, en ese camino hay que irse enlazando con las distintas organizaciones que piensan sobre la inclusión educativa, así, la Federación Internacional Fe y Alegría, en su plan de prioridades establece, atendiendo al $4^{\circ}$ objetivo de Desarrollo sostenible que la Asamblea General de las Naciones Unidas aprobó en septiembre de 2015 para los próximos 15 años, "Garantizar una educación inclusiva, equitativa y de calidad y promover oportunidades de aprendizaje permanente para todas y todos", lo que representa para Fe y Alegría Ecuador un espacio para proponer innovación educativa. Esto no solamente se refiere a la atención a las personas en situación de discapacidad, sino a la valoración de la diversidad cultural, étnica, religiosa, social, de modos de aprender, de capacidades, de género, entre otras, como un enriquecimiento de la comunidad educativa y de la realidad en pleno (Federación Internacional Fe y Alegría, 2013, pág 1). 
Así comprendida la educación, requiere de una transformación profunda de mentalidad e imaginarios que incidan en un cambio de actitudes y acciones concretas, en un cambio de metodologías, sistemas de enseñanza, infraestructura y comunidades educativas que respondan a la diversidad. Los distintos actores tienen que dirigir su mirada hacia ese cambio paulatino, donde la sociedad es de vital importancia para repensar y aportar; pero el papel más relevante lo tiene el espacio vital en el que se socializa e interactúa en diversidad, donde existe un intercambio de saberes, experiencias y vivencias, que va provocando inquietudes y movilizando conciencias para generar valores, actitudes y un sentido común que garanticen una mayor comprensión sobre esta problemática y direccione la inclusión educativa como meta de transformación social.

\section{Educación Popular e Inclusión Educativa}

Hablar de inclusión desde la educación popular es promover, provocar, implicar e implicarse en el afán de minimizar las posibles barreras que causan situaciones de exclusión y que limitan la posibilidad de que todas las personas tengan una vida plena y digna de acuerdo a sus potenciales y no a sus debilidades.

Fe y Alegría concibe a la educación ligada a los procesos sociales, considera vital partir de la lectura y relectura de los diferentes contextos y escenarios en los que se desarrolla la práctica educativa para visualizar con una mirada más profunda las problemáticas sociales, las necesidades educativas y los intereses de la población excluida y, al mismo tiempo, para plantear alternativas desde la reacción y reflexión, en pos de llegar a aquella conciencia que posibilite la transformación social, educativa, familiar y cultural.

Para que esto suceda es indispensable el compromiso de la sociedad civil en la incidencia sobre las políticas públicas. Para Fe y Alegría si la incidencia no fortalece a la sociedad civil, no haría otra cosa que reproducir el modelo excluyente (Sarmiento, 2015). 
Para hacer posible esta transformación se piensa a la educación sostenida en cuatro pilares (Fe y Alegría, 2016):

- Pilar ético: da cuenta de la opción radical por la búsqueda de la transformación, al considerarla un imperativo categórico respecto de la sociedad inequitativa y desigual de la que formamos parte, y al concebirla como un objetivo ulterior que permita vincularnos con el futuro de manera corresponsable.

- Pilar epistemológico: tiene que ver con el conocimiento, con la esencia del hecho educativo y con la idea de que cada uno posee un saber particular que tiene valor como tal y que requiere ser considerado en los procesos educativos.

- Pilar pedagógico y metodológico: remite a las maneras en que se llevan adelante los procesos educativos desde una perspectiva de diálogo de saberes orientada a la innovación.

- Pilar político: implica "tomar postura" no desde el punto de vista partidista, sino desde el rol que cada persona asume el mundo, viendo a la educación como un verdadero acto político orientado al bien común.

Dichos pilares son fundamentales para consolidar una educación de calidad, una educación para todas y todos. $Y$, si se toma en cuenta que el foco de la inclusión educativa es la transformación desde la innovación y reflexión de las prácticas educativas, de las relaciones sociales, familiares y comunitarias, los pilares se constituyen en el andamiaje.

En la comprensión del ser y hacer de Fe y Alegría y en su apuesta por la inclusión es necesario abordar los conceptos de promoción social, educación de calidad y la educación inclusiva.

\section{Intencionalidad de Promoción social}

Tiene directa relación con la inclusión educativa y la educación inclusiva, puesto que la atención a la diversidad no es asistencialista, más bien, nos llama a movilizarnos, a inquietarnos, a reaccionar desde cada indivi- 
dualidad para generar cambios colectivos; al mismo tiempo tiene relación con la educación popular "puesto que ambos se intercomunican e interactúan en dos escenarios entrelazados, identificados como comunidad escolar (la escuela) y comunidad educativa (su contexto inmediato)" (F.I. Fe y Alegría, 2007 citado en Fe y Alegría, 2016, págs. 46-47)

Desde la intencionalidad de promoción social, se mira con claridad que la inclusión educativa ocupa un lugar importante en la educación popular, ayuda además a educar sujetos íntegros e integrales que cuiden de sí mismos y de la otra persona, que se apasionen por la humanidad estimulando el crecimiento personal y comunitario.

La atención desde edades tempranas para el fortalecimiento de las áreas del desarrollo evolutivo contribuye a la mejora de las condiciones de la calidad de vida; mediante un trabajo preventivo, equitativo y responsable, garantiza la participación, la convivencia, el aprendizaje y la permanencia en el centro educativo de niños, niñas, adolescentes, educadores y familias.

\section{Educación de calidad}

Se define a la educación de calidad como un proceso que promueva el progreso de los estudiantes, implantando prácticas educativas que potencian los logros intelectuales, morales, sociales y emocionales, teniendo en cuenta su nivel socioeconómico, su medio familiar y su aprendizaje previo (Federación Internacional Fe y Alegría, 2009, pág. 25). Se establece así una concordancia con la concepción de educación inclusiva y la de calidad:

- Ser accesible a todos los ciudadanos, en concordancia con el derecho a la educación.

- Facilitar los recursos personales, organizativos y materiales ajustados a las necesidades de los estudiantes para proveer las condiciones necesarias que les permitan tener oportunidades de progreso académico y personal; acciones que deben darse en sintonía con la gestión de los equipos directivos y con las dimensiones administrativa, organizacional, pedagógica, comunitaria convivencial y específica del contexto. Esta última debe tener especial énfasis en casos parti- 
culares para poder dinamizar acciones en beneficio de la población estudiantil.

- Generar cambios e innovación a nivel de centro educativo y en las aulas, proceso que tiene relación con la innovación educativa que promueve Fe y Alegría - Ecuador en la que tiene lugar la inclusión educativa, dada su relación con el currículo que debe cumplirse, un currículo inclusivo, crítico y transformador. Dicho currículo va direccionado hacia la atención a la diversidad, enfatizando en la diversidad funcional, denominada también en los contextos educativos como Necesidades Educativas Especiales asociadas a la discapacidad, o con un término más asertivo adoptado hoy en día: diversidad funcional. A la diversidad funcional a su vez se la concibe hoy como una situación social del desarrollo en relación a los derechos humanos, más que como una problemática individual.

- Promover la participación activa de las y los estudiantes tanto en el proceso de aprendizaje como en la vida del centro educativo en el marco de los valores, en donde todos y todas se sientan respetadas y valoradas, proceso que tiene lugar a medida que se va desarrollando el fortalecimiento de una comunidad inclusiva a través de la evolución del lenguaje positivo y de la conciencia y la aplicación de los valores institucionales de Fe y Alegría-Ecuador.

- Lograr la participación de las familias en la vida del centro educativo e insertarse en la comunidad, lo que desde Fe y Alegría se promueve a partir del diálogo de saberes y a partir de la integración de las familias en el proceso educativo desde el nivel inicial especializado y ordinario para construir en conjunto ciudadanía y convivencia pacífica.

- Estimular y facilitar el desarrollo y bienestar de los educadores y las educadoras, relacionado con la gestión y liderazgo pedagógico de $\mathrm{Fe}$ y Alegría-Ecuador.

En correspondencia a lo mencionado, el criterio de calidad se aborda también desde la satisfacción de necesidades, considerada como una dimensión y como una prioridad universal para la mejora de la calidad de vida, procurando que sea una vida con dignidad y subra- 
yando la importancia de garantizar la equiparación de oportunidades; resaltando a la vez el valor a las personas que forman parte de los grupos minoritarios, su reconocimiento y respeto por toda la comunidad educativa, pero sobre todo procurado que su autovaloración y autoimagen sea positiva.

\section{De la Inclusión Educativa a la Educación Inclusiva}

Para Fe y Alegría- Ecuador el sentido de la inclusión educativa radica en el ejercicio de los derechos humanos, planteamiento que ayuda a fortalecer una sociedad que no discrimine y que encuentre en cada persona potencialidades, reconociendo y valorando la diferencia. Asimismo, parte de una equiparación de oportunidades en todos los espacios para garantizar la socialización, recreación, participación y toma de decisiones, busca erradicar la exclusión educativa y social identificando las barreras que limitan el proceso de inclusión y, por lo tanto, apunta a prevenir la marginalidad y promover la formación humana desde una educación humanizadora e innovadora.

Enfatiza en la inclusión de las personas con diversidad funcional (discapacidad), con un criterio que parte de que la limitación de las personas no proviene de su condición individual, sino de la falta de oportunidades, que se convierte en una barrera para la adquisición de autonomía, el aprendizaje, la participación y el sentido de pertenencia a la comunidad, necesarios para desarrollar sus capacidades y habilidades.

Toma en cuenta también las necesidades educativas no asociadas a la diversidad funcional, con el mismo sentido, lo que se hace posible desde la atención a la diversidad.

Esta comprensión mucho más social, histórica y cultural, se sustenta además en la visión espiritual. Es necesario relacionarnos con personas diferentes, de distintas creencias, culturas, países, ideologías, clases sociales, capacidades; transformando y transformándonos desde el vínculo cercano con personas diferentes, pero también con semejanzas, de manera que vivamos la diversidad.

La concepción de Educación Inclusiva, parte de la conferencia internacional de educación de 2008 de UNESCO, desde un enfoque 
integrador, holístico, que posibilita el acceso, calidad, equidad y calidez, a los niños, niñas y jóvenes sin distinción de ninguna naturaleza, ni discriminación de ningún tipo, sea racial, económica, cultural, étnica, religiosa y/o con capacidades diferentes o especiales. En palabras de Rosa Blanco la Educación Inclusiva se fundamenta en principios éticos, sociales, económicos y educativos. "Es el medio para hacer efectivo el derecho a una educación de calidad sin discriminaciones y en igualdad de oportunidades" (UNESCO, 2008, pág. 9).

A ello se añade desde Fe y Alegría (2016) el objetivo de inclusión educativa señalado en el Horizonte Pedagógico Pastoral que postula lo siguiente: "brindar respuestas apropiadas al amplio espectro de necesidades de aprendizaje tanto en entornos formales como no formales de la educación." (Pág. 154) Y, en cuanto a su función, agrego lo siguiente: "El propósito de la educación inclusiva es permitir que las y los maestros y estudiantes se sientan cómodos ante la diversidad y la perciban no como un problema, sino como un desafío y una oportunidad para enriquecer el entorno de aprendizaje" (UNESCO, 2005, pág. 14).

Para concretar la teoría y el ideal, se hace necesario educar desde la inclusión (Federaciónlnternacional de Fe y Alegría, 2013) para ir hacia la construcción de una educación de calidad; para lo cual Fe y Alegría se plantea los siguientes desafíos:

- Optar por las personas excluidas con la convicción de atender a las más vulnerables.

- Establecer políticas inclusivas en los centros educativos para eliminar los mecanismos de excluyentes.

- Identificar las barreras que limitan e impiden al acceso a la educación, al aprendizaje, a la participación y a la promoción y culminación de estudios de las personas con diversidad funcional y /o que requieren atención prioritaria.

- Promover el desarrollo integral de todos los estudiantes y sus familias. 
- Revisar el currículo, el cual suele ser causa de exclusión por no tener en cuenta las especificidades de los contextos y las personas.

- Reconocer la diversidad promoviendo el desarrollo de habilidades sociales y de aprendizaje entre pares y en grupos cooperativos.

- Incidir políticamente para que el gobierno competente reflexione sobre la inversión económica y de talento humano que haga posible una atención de calidad a la diversidad.

- Promover una sociedad sensible que reaccione ante las injusticias.

- Continuar formando a los distintos actores educativos para que se posibilite la transformación educativa y social que integren la pedagogía, la fe y la justicia y dote de sentido las vidas (Federación Internacional Fe y Alegría, 2016).

Como se puede ver, la inclusión educativa se considera como un eje nuclear, que se articula desde las declaraciones estratégicas, planificación estratégica, organizacional y operativa, lo que otorga valor a la oferta educativa del Movimiento. Para Fe y Alegría, hablar de inclusión es pasión, es audacia, es sabiduría, es movilizarse, es inquietarse, es reaccionar, es denunciar, es un estilo de vida, jes conciencia individual, jes conciencia colectiva!

\section{Bibliografía:}

García, B. (2010) Pensamiento de Simón Rodríguez: La educación como proyecto de inclusión social. Revista Colombiana de educación. Universidad Colombiana de Caldas. Bogotá Colombia.

Federación Internacional Fe y Alegría (2009) El Sistema de Mejora de la Calidad en Fe y Alegría. Una Mirada desde la Educación Popular. 
Federación Internacional de Fe y Alegría (2013) La educación Inclusiva y sus desafíos. Revista Internacional de Fe y Alegría $N^{\circ} 14$.

Federación Internacional Fe y Alegría (2016) Plan de Prioridades Federativas 2016-2020.

Fe y Alegría Ecuador (2016) Estatuto Orgánico de Gestión Organizacional por procesos.

Fe y Alegría Ecuador (2016) Horizonte Pedagógico Pastoral.

Plan Apostólico de la Provincia Ecuatoriana de la Compañía de Jesús. 2017-2020

FederaciónInternacional Fe y Alegría (2013). Educación Inclusiva en la Misión de Fe y Alegría. Documento XLIV Congreso Internacional. Brasil.

Rodríguez, S. (1990). Sociedades americanas (Vol. 150) Fundación Biblioteca Ayacucho.

Sarmiento, Jaime. Tesis: La contribución de la sociedad civil en las políticas locales de inclusión educativa, el caso de Fe y Alegría en el periodo 2011-2012. Abril 2015. 